\title{
Correction to: Convergence of vertex-reinforced jump processes to an extension of the supersymmetric hyperbolic nonlinear sigma model
}

\author{
Franz Merkl ${ }^{1}$ - Silke W. W. Rolles ${ }^{2}$ - Pierre Tarrès ${ }^{3,4}$ \\ Published online: 26 February 2020 \\ (c) Springer-Verlag GmbH Germany, part of Springer Nature 2020
}

\section{Correction to: Probability Theory and Related Fields (2019) 173:1349-1387 https://doi.org/10.1007/s00440-018-0855-8}

In the original publication, "Acknowledgements" section was incorrect. The correct "Acknowledgements" is given below.

Acknowledgements The authors would like to thank an anonymous referee and the associate editor for very constructive comments, helping us to improve the paper. This work is supported by the National Science Foundation of China (NSFC), Grant No. 11771293, and by the Agence Nationale de la Recherche (ANR) in France, Project MALIN, No. ANR-16-CE93-0003.

Publisher's Note Springer Nature remains neutral with regard to jurisdictional claims in published maps and institutional affiliations.

The original article can be found online at https://doi.org/10.1007/s00440-018-0855-8.

Silke W. W. Rolles

merkl@math.lmu.de

Franz Merkl

srolles@ma.tum.de

Pierre Tarrès

tarres@nyu.edu

1 Mathematical Institute, Ludwig-Maximilians-Universität München, Theresienstr. 39, 80333 Munich, Germany

2 Zentrum Mathematik, Bereich M5, Technische Universität München, 85747 Garching bei München, Germany

3 NYU-ECNU Institute of Mathematical Sciences at NYU Shanghai, Courant Institute of Mathematical Sciences, New York, NY 10012, USA

4 CNRS \& CEREMADE, Université Paris-Dauphine, PSL Research University, Place de Lattre de Tassigny, 75016 Paris, France 